\title{
On the Modeling of the Diffraction Pattern from Metal Nanocrystals
}

\author{
LUCA GELISIO and PAOLO SCARDI
}

The powder diffraction patterns of spherical nanocrystals made of five different fcc metals were generated using atomistic models within a Molecular Dynamics simulation. Static and dynamic effects are interpreted and discussed within the framework of two different approaches, respectively, based on (1) a Reciprocal Space and (2) a Direct Space representation of diffraction. Chosen elements display a wide range of properties, especially related to material stiffness and elastic anisotropy, so to deeply challenge interpretation paradigms. The effect of the shape on static and dynamic features is also addressed.

DOI: $10.1007 / \mathrm{s} 11661-014-2407-\mathrm{x}$

(C) The Minerals, Metals \& Materials Society and ASM International 2014

\section{INTRODUCTION}

Metal nanocrystals are a subject of study in several and quite different research fields, like clean energy production and biomedical applications, both requiring nanocrystalline metal surfaces to activate/ enhance oxidation. ${ }^{[1,2]}$ In both cases, the key is controlling nanocrystal size and shape, to obtain a specific catalytic behavior and improve the performance. While nanotechnology actively pursues these important achievements, characterization techniques need to evolve to provide increasingly detailed information. Powder diffraction has so far lagged behind, as in many cases even in top level research studies just qualitative or partial information has been exploited: X-Ray Diffraction (XRD) line profiles are often analyzed in terms of peak width for a quick assessment of some characteristic length of the studied system. ${ }^{[3,4]}$ Well known alternatives, much exploited in the cited studies, employ Transmission Electron Microscopy (TEM): spectacular pictures and detailed information can be obtained by high resolution TEM, and more specifically by HighAngle Annular Dark-Field imaging (HAADF), ${ }^{[5-7]}$ although the analysis can hardly concern more than just a few nanoparticles. Sample preparation can result in a biased sampling of the statistical population (e.g., by excluding larger or smaller particles, or by focusing only on loose items and excluding agglomerates), and beam energy can degrade the specimen or promote phase transformations. XRD is a perfectly complementary technique, as it can support electron microscopy in providing a sound statistical basis: a typical powder diffraction analysis involves millions to billions of crystalline domains. However, much is still to be understood on the XRD from nanocrystalline materials.

LUCA GELISIO, Ph.D. Student, and PAOLO SCARDI, Professor, are with the Department of Civil, Environmental and Mechanical Engineering, University of Trento, via Mesiano 77, 38123 Trento, Italy. Contact e-mail: paolo.scardi@unitn.it

Manuscript submitted April 1, 2014.

Article published online June 21, 2014
As shown in this paper, the modern powder diffraction theory provides much better and more refined methods than the nearly centenary Scherrer equation and related integral breadth methods. ${ }^{[8,9]}$ Methodologies proposed over the past decade evolved following two different schools of thought. According to the traditional paradigm (1), diffraction is studied in Reciprocal Space (RS); (a) diffraction peaks are described by suitable profile functions, just flexible ones (as in traditional profile fitting), or (b) model-based profile functions, for a direct evaluation of (nano)structural parameters. ${ }^{[10]}$ As an alternative, (2) the Debye Scattering Equation (DSE) is based on the Direct Space (DS) representation of nanocrystals. This provides a detailed picture of the nanostructure, possibly down to the atomic level, although to the cost of a higher complexity and computational demand. ${ }^{[11-13]}$

Differences between the two approaches have not been fully investigated so far, also because no simple and clear experimental cases are available for such a comparison. Atomistic models are convenient in this context: Molecular Dynamics (MD) can be used to build model systems of metal nanocrystals, then generate a corresponding powder diffraction pattern to be used as a plausible benchmark to compare RS and DS methods. ${ }^{[14]}$

Besides comparing the two methods, the present work shows some peculiarities and information that can be provided by powder diffraction on the surface relaxation effect which dominates most properties of metal nanoparticles. Several metals and domain sizes are compared to further highlight differences arising from different basic properties of the metal nanocrystals.

\section{EXPERIMENTAL AND METHODS}

\section{A. Generation of Metal Nanocrystals and Powder Patterns}

Atomistic models of spherical nanocrystals were built for several fcc metallic elements (Table I) and the $\operatorname{DSE}^{[15,16]}$ used to generate the powder diffraction 
Table I. Lattice Parameter (a), Young modulus projected along different directions $(E)$, Zener Ratio $\left[\mathrm{Zr}=2 C_{44} /\left(C_{11}-C_{12}\right)\right]$, Surface Energies $(\gamma)$ and Melting Temperature $\left(T_{m}\right)$ for Investigated Elements

\begin{tabular}{|c|c|c|c|c|c|}
\hline & $a(\AA)$ & $E_{100}(\mathrm{GPa})$ & $E_{110}(\mathrm{GPa})$ & $E_{111}(\mathrm{GPa})$ & $E(\mathrm{GPa})$ \\
\hline $\mathrm{Rh}$ & 3.8034 & 300.6 & 405.5 & 458.9 & 369 \\
\hline $\mathrm{Pd}$ & 3.8900 & 78.9 & 150.6 & 216.2 & 127 \\
\hline $\mathrm{Ag}$ & 4.0900 & 48.5 & 92.3 & 132.2 & 78 \\
\hline $\mathrm{Pt}$ & 3.9200 & 136.3 & 186.0 & 211.8 & 180 \\
\hline \multirow[t]{2}{*}{$\mathrm{Pb}$} & 4.9508 & 11.8 & 25.3 & 41.0 & 25 \\
\hline & $\mathrm{Zr}$ & $\gamma_{100}\left(\mathrm{Jm}^{-2}\right)$ & $\gamma_{110}(\mathrm{GPa})$ & $\gamma_{111}(\mathrm{GPa})$ & $T_{\mathrm{m}}(\mathrm{K})$ \\
\hline $\mathrm{Rh}$ & 1.651 & 2.481 & 2.381 & 2.233 & 2065 \\
\hline $\mathrm{Pd}$ & 2.982 & 1.645 & 1.747 & 1.529 & 1680 \\
\hline $\mathrm{Ag}$ & 3.000 & 1.042 & 1.125 & 0.977 & 1255 \\
\hline $\mathrm{Pt}$ & 1.604 & 1.778 & 1.934 & 1.694 & 1890 \\
\hline $\mathrm{Pb}$ & 3.753 & 0.476 & 0.532 & 0.405 & 680 \\
\hline
\end{tabular}

Properties are calculated using values taken from Ref. [21].

pattern. ${ }^{[13,17]}$ Particles were carved out of an ideal fcc lattice, removing atoms with less than six nearest neighbors (as-built objects hereinafter). Equilibrium atomic positions and thermal vibrations were obtained by MD, using the Large-scale Atomic/Molecular Massively Parallel Simulator (LAMMPS ${ }^{[18]}$ ), with atomic interaction based on the Embedded Atom Method $\left(\mathrm{EAM}^{[19-21]}\right)$. Integration timestep was set to one hundredth of the reciprocal of the largest phonon frequency at room temperature $(R T=298.150 \mathrm{~K})$. The Temperature ( $T$ ) was initially set to $447.225 \mathrm{~K}$ and then lowered to RT. At a later stage, the constraint on $\mathrm{T}$ was removed and the total Energy $(E)$ kept constant (NVE ensemble). The Number of atoms (N) and the Volume (V) were also kept constant during the simulation, which is summarized in Figure 1. In the last NVE region (i.e., microcanonical ensemble: constant $\mathrm{N}, \mathrm{V}$ and $\mathrm{E}$ ) atomic coordinates were dumped every 1000 steps along the MD trajectory (250 frames). From these data, two different samples were obtained: (1) the DSE was applied to atomic coordinates at each snapshot, and the time-averaged XRPD pattern computed (time-averaged); (2) the DSE was applied to the time-averaged atomic positions (space-averaged). The time-averaged XRPD pattern should mimic a real experiment, being both static (atomic arrangement) and dynamic (atomic vibrations) features included, whereas the space-averaged sample is meant to leave out the effect of vibrations, being the DSE only applied to the average atomic positions, so to single-out the effect of atomic displacement due to finite size of the object.

Static features were assessed through the displacement field,

$$
\Delta_{i}=-\left(\mathbf{r}_{i}^{\mathrm{sa}}-\mathbf{r}_{i}^{\mathrm{ab}}\right),
$$

where $\mathbf{r}_{i}^{\mathrm{ab}}$ is the vector connecting atom $i$ to the reference frame in the as-built configuration and $\mathbf{r}_{i}^{\mathrm{sa}}=\left\langle\mathbf{r}_{i}(\mathrm{t})\right\rangle_{t}$ in the space-averaged one (the symbol \langle\rangle$_{t}$ denotes the average over time, i.e., the entire MD trajectory).

Moreover, a simple expression for the average atomistic strain can be built computing the difference of bond length $\left(\left|r_{i j}\right|, j\right.$ being a nearest neighbor of $\left.i\right)$,

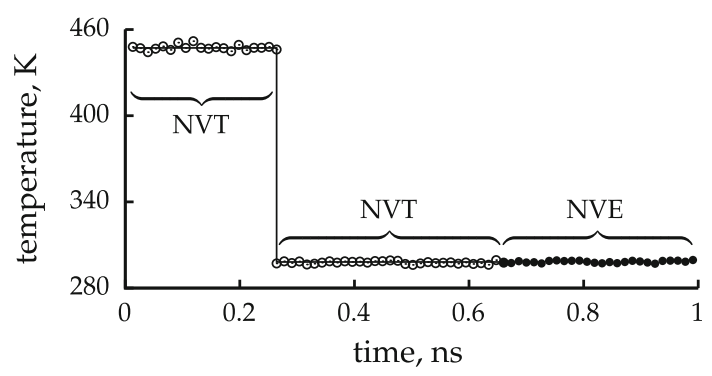

Fig. 1-Schematic representation of the MD simulation procedure for the 6 unit cell radius $\mathrm{Pb}$ sphere, with indication of the different stages until the atomic coordinates are extracted (NVE ensemble) and used in the generation of nanocrystal powder diffraction pattern (see text for details).

$$
\varepsilon_{i, Z}=\frac{1}{Z} \sum_{j=1}^{z}\left(\frac{r_{i j}^{\mathrm{sa}}}{r_{i j}^{\mathrm{ab}}}-1\right)
$$

and averaging the information over the $Z$ nearest neighbors. Atomic vibrations were represented by the traditional temperature factor ( $B$-factor, in the following, $B_{\text {iso }}$ ), which appears in the Debye-Waller factor

$$
B_{\text {iso }}=\frac{8 \pi^{2}}{3}\left\langle\frac{1}{N} \sum_{i=1}^{N}\left|\mathbf{r}_{i}(t)-\mathbf{r}_{i}^{\mathrm{sa}}\right|^{2}\right\rangle_{t}=\frac{8 \pi^{2}}{3}\langle\operatorname{MSD}(t)\rangle_{t}
$$

i.e., the time-average of the Mean Squared Displacement (MSD). In turn, this quantity expresses the squared difference of the vector $\left(\mathbf{r}_{i}^{\text {sa }}\right)$ connecting the atom $i$ in the reference configuration (which, in this case, is space-averaged, as atoms oscillate around these positions) and the same vector at a given time $t$.

\section{B. Whole Powder Pattern Modeling (WPPM) and DSE}

WPPM is a perturbation approach applied to a perfect crystal model in RS (see Reference 10 and references therein). Each peak profile in a powder pattern is represented as a convolution of effects 
originating from the microstructure, here defined as any deviation from an otherwise perfect crystal lattice. Each effect has a corresponding line profile, usually known in a simple analytical form, function of a few physically sound parameters. Among the most frequently implemented models, finite size and shape of the crystalline domain, dislocations of different type and stacking faults. ${ }^{[10,22,23]}$ The key to use a convolution of effects is to exploit the convolution theorem for Fourier Transforms, which turns a computationally complex problem of folding into a simple multiplication of different terms. As of the most recent developments, ${ }^{[24]}$ WPPM can use virtually any crystalline domain shape and strain models for dislocations in any crystal system. ${ }^{[25]}$ Nanocrystals can then be studied down to small sizes, with the highest precision for the spherical domain shape. ${ }^{[26]}$ Less accurate is the description of the complex effects caused by the nanocrystal surface, which displaces atoms from the expected perfect crystal positions: so far only simplified models for this surface relaxation effect could be implemented in a WPPM procedure. ${ }^{[27]}$ Thermal effects can also be handled by WPPM, according to a model originally proposed by Warren ${ }^{[16,28]}$ and recently adapted to the finite size of nanocrystals. ${ }^{[29]}$

In the following, we use a spherical nanocrystal model, including Debye-Waller factor and Thermal Diffuse Scattering (TDS) for the spherical shape. ${ }^{[24]}$ The complex and anisotropic atomic displacement caused by surface relaxation is treated in a simplified way, providing for a shift of the average unit cell parameter and a flexible, although not entirely rigorous, r.m.s. atomic displacement (microstrain). Its peak profile component is represented by a pseudo-Voigt function, ${ }^{[30]}$ whose peak width is allowed to vary for different $(h k l) \mathrm{s}$ according to the invariant form for cubic systems ${ }^{[31]}$

$$
A+B H=A+B \frac{h^{2} k^{2}+k^{2} l^{2}+l^{2} h^{2}}{\left(h^{2}+k^{2}+l^{2}\right)^{2}} .
$$

Peak positions are corrected in a similar way, by allowing interplanar distances, $d_{h k l}$, to vary according to a similar invariant factor $\left(d_{h k l}\right.$ is multiplied by $\left.A^{\prime}+B^{\prime} H\right)$. As shown in more detail below, this approach provides useful information, even though an accurate description necessarily requires an atomistic model and DS approach. ${ }^{[14,32]}$

In this framework, the DSE expresses (within the kinematical approximation) the intensity scattered from a powder of identical atomic aggregates composed of $N$ atoms connected by vectors $r_{i j}$ and scattering with a (spherically symmetric) form factor $f_{i}$, $\left.15-17,33\right]$

$$
I(q)=\sum_{i=1}^{N} \sum_{j=1}^{N} f_{i}(q) f_{j}(q) \operatorname{sinc}\left(q r_{i j}\right)
$$

It is the contribution of each atomic pair which is computed, so the XRPD pattern can be generated for any atomic arrangement. As a case of study, the pattern of selected space-averaged particles were modeled using the reference as-built object as starting configuration, and a model adapted from the one proposed in Reference 14 to displace atoms and account for lattice relaxation,

$$
\begin{aligned}
& \Delta_{i}\left(l, \alpha, a, \sigma ; h, k, l, Z_{i}, \mathbf{r}_{i}^{\mathrm{ab}}\right) \\
& \quad=\left(\mathbf{r}_{i}-\mathbf{r}_{i}^{\mathrm{ab}}\right)=\kappa(h, k, l)\left[\left(1-\frac{a}{a^{\mathrm{ab}}}\right)+\sigma \ln \left(\frac{Z_{i}}{12}\right)\right] \mathbf{r}_{i}^{\mathrm{ab}} \\
& \kappa(h, k, l)=\left\{l S_{11}-2 \alpha\left[S_{11}-S_{12}-\frac{S_{44}}{2}\right] \frac{h^{2} l^{2}+l^{2} k^{2}+k^{2} h^{2}}{\left(h^{2}+k^{2}+l^{2}\right)^{2}}\right\} .
\end{aligned}
$$

A strain localized on the surface $\left(Z_{i} \neq 12\right)$ is added to a strain constant through the particle, $\chi=\left(1-a / a^{\mathrm{ab}}\right)$. These quantities are, in turn, mapped onto the material through the elastic tensor, described by the constant coefficients $S_{\mathrm{nm}}$. The displacement of a given atom from its reference position is then projected on the radial versor so to obtain a radial deformation $\left(\varepsilon_{i, \mathrm{r}}=\Delta_{i} / r_{i}^{\mathrm{ab}}\right)$, suitable for the spherical symmetry of test cases.

\section{RESULTS AND DISCUSSION}

The statistical quality of each fit is assessed through a Normalized Residual Sum of Squares (NRSS),

$$
\mathrm{NRSS}=\frac{1}{Q} \sum_{q=1}^{Q}\left[\frac{I_{\mathrm{s}}(q)-I_{\mathrm{m}}(q)}{N f^{2}(q)}\right]^{2}=\frac{1}{Q} \sum_{q=1}^{Q}\left[\Im_{\mathrm{s}}(q)-\Im_{\mathrm{m}}(q)\right]^{2} .
$$

The calculated intensity (Eq. [5]) for both the simulated object $\left(I_{\mathrm{s}}\right)$ and the model $\left(I_{\mathrm{m}}\right)$ is normalized over (1) the number of atoms $(N)$ and (2) the squared atomic form factor. This way, (1) particles composed by a different number of scatterers or (2) different chemical elements can be compared: the residual sum of squares of the two interference functions $\left(\Im=I / N f^{2}\right)$ is computed. Finally, the fact that patterns may have been sampled with a different number of points $(Q)$ is also taken into account.

As-built nanocrystals give a nearly perfect match between RS and DS approach (e.g., see Figure 2). Just two parameters were refined by WPPM: unit cell parameter, $a$, and domain diameter, $D$, while all other parameters were fixed, including the scale factor calculated from the known number of atoms. Refined $a$ agrees with the model better than $4 \times 10^{-6}$, and in some cases (larger sizes) better than $1 \times 10^{-6}$. Even little differences can be explained, considering the powder pattern was generated by the DSE for a single sphere model whereas the RS approach, as implemented by WPPM, implicitly assumes the conditions of Ino and Minami ${ }^{[26,34]}$ : an average is made of patterns of spherical models centered in different positions, which corresponds to "carve" slightly different spheres out of a perfect (infinite) lattice. Differences usually concern just a fraction of atoms on the surface, which become significant at the nanoscale. ${ }^{[26]}$ 

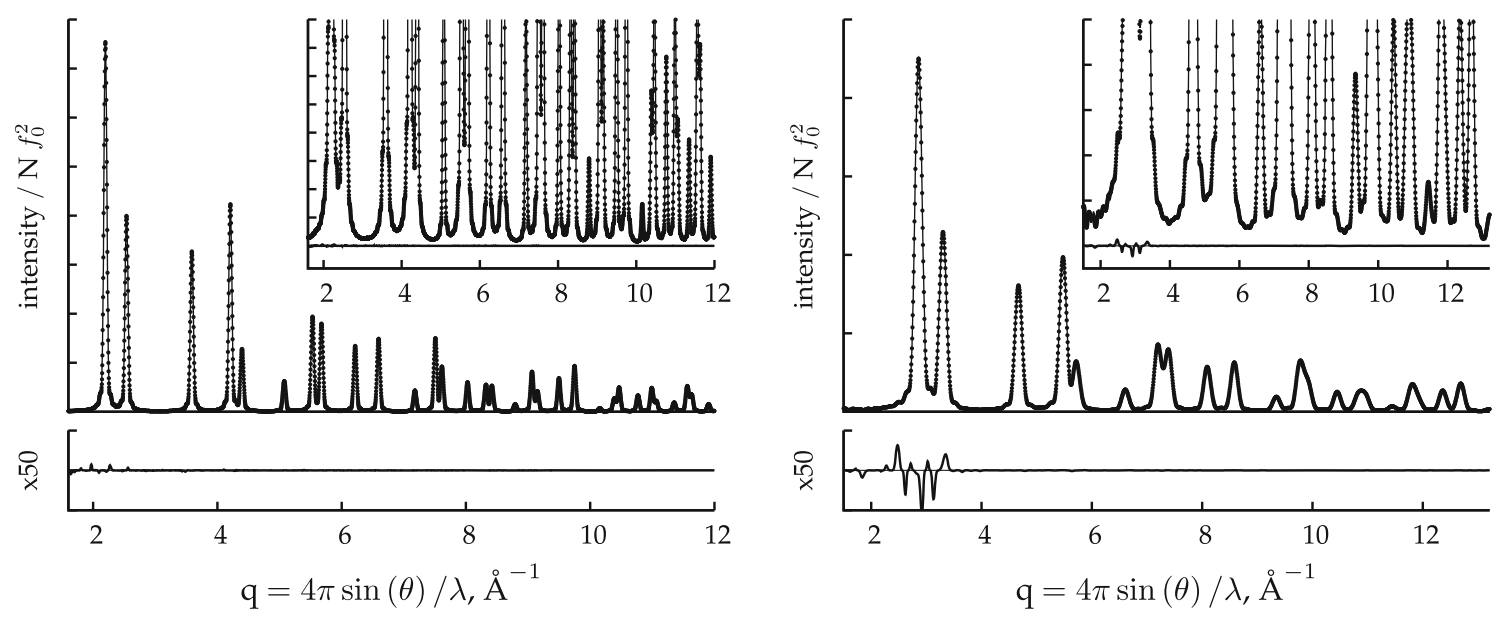

Fig. 2-WPPM results (RS modeling) for the powder patterns of MD simulated spheres of 12 (28867 atoms) and 6 (3559 atoms) unit cells, respectively, for $\mathrm{Pb}\left(\mathrm{left} ; N R S S=1.9 \times 10^{-15}\right.$ ) and $\mathrm{Rh}$ (right; $N R S S=2.1 \times 10^{-12}$ ). The inset shows a detail of the peak tail region and fine discrepancies in the residual (difference between data and model, line below).

WPPM of the powder pattern for MD simulated spheres (time-averaged XRPD pattern), although not as satisfactory as for the corresponding as-built spheres, provides interesting results. Just six parameters were allowed to vary during the WPPM: besides $a$ and $D$, Debye-Waller $B_{\text {iso }}$ factor, effective microstrain with anisotropy parameter $B$, and effective macrostrain anisotropy parameter $B^{\prime}$ (see Section III).

One of the most evident effects is the shrinkage of the metal nanoparticles caused by surface tension, which in turn is a consequence of the lower coordination of surface metal atoms. This effect is promptly seen in terms of domain size, but is also evident from Figure 3, showing the relative change of unit cell parameter (with respect to the as-built, starting value) for the five studied metals and four different spherical domain diameters. The effect decreases progressively for increasing size, toward the "bulk" value (zero), although each trend depends on the specific metal: as a general tendency, the effect is stronger for softer (lower Young modulus) metals, so the smallest effect is for $\mathrm{Rh}$, the largest for $\mathrm{Pb}$.

Domain size also affects the Debye-Waller factor, as a result of the decreased number of atoms in surface regions. ${ }^{[35-38]}$ The refined value of $B_{\text {iso }}$, which is inversely proportional to the Young modulus of the studied fcc metals (Figure 4(a)), increases in smaller domains (Figure 4(b)). As shown in Figure 4(a), values refined by WPPM are in a reasonably good agreement with the reference $B_{\text {iso }}$ given by MD, as obtained from the atomic coordinates along the MD trajectory (Eq. [3]).

Lines in Figure 4(b) represent $B_{\text {iso }}$ calculated for a $40 \times 40 \times 40$ unit cell cube, implementing Periodic Boundary Conditions (PBCs) in the MD, so to mimic an "infinite" crystal. Even if the periodicity condition alters the vibrational properties (the longest phononic wavelength is proportional to the size of the simulation domain), values are in good agreement with the literature, ${ }^{[39]}$ and correctly point out the asymptotic trend of $B_{\text {iso }}$ with the increasing nanocrystal size.

The discrepancy between the WPPM result and $B_{\text {iso }}$ given by MD, once again, can only partly be attributed

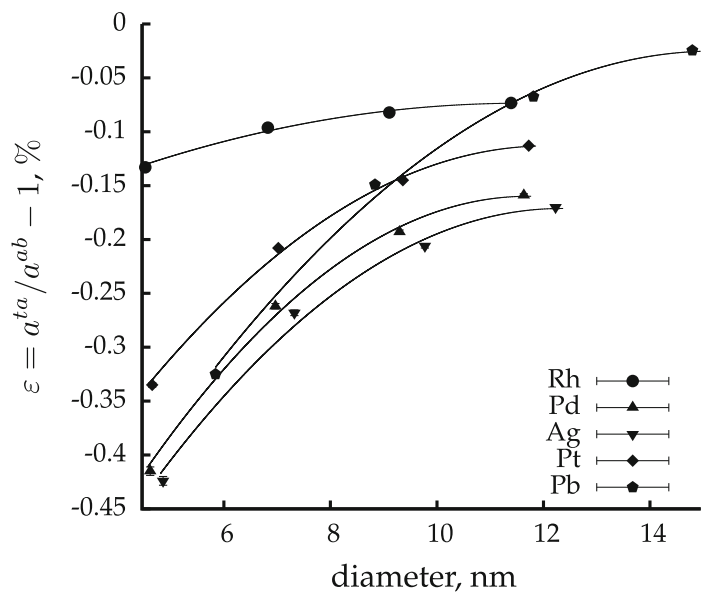

Fig. 3-WPPM results for the relative change of unit cell parameter after MD simulation (time-averaged, $t a$ ) as a function of the spherical domain diameter for the five studied fcc metals. Parabolic trends are drawn just to drive the eye.

to limitations in the RS approach, as it mostly stems from the different way to measure the underlying property, which is the Mean Squared Displacement (MSD). While the MSD (and in turn, $B_{\text {iso }}$ ) from MD is an arithmetic average over each atom (see Section III), the Debye-Waller factor refined by WPPM mostly depends on the depression of Bragg peak intensity with increasing scattering vector, and to some extent on the Temperature Diffuse Scattering. Moreover, as shown by MD simulations (Figure 5), the amplitude of vibrations of atoms sitting on the surface is much larger, being their coordination lower than the bulk value, a feature clearly responsible for the dependency of the mean $B_{\text {iso }}$ value with the size (Figure 4(b)).

The role of the temperature diffuse scattering term is addressed in Figure 6, where the powder pattern for silver and lead, considering spherical domains with 6 or 12 unit cell radius is presented. Both the modeling results with a realistic TDS model (right), ${ }^{[29]}$ and by using the so-called Debye TDS, i.e., the diffuse 

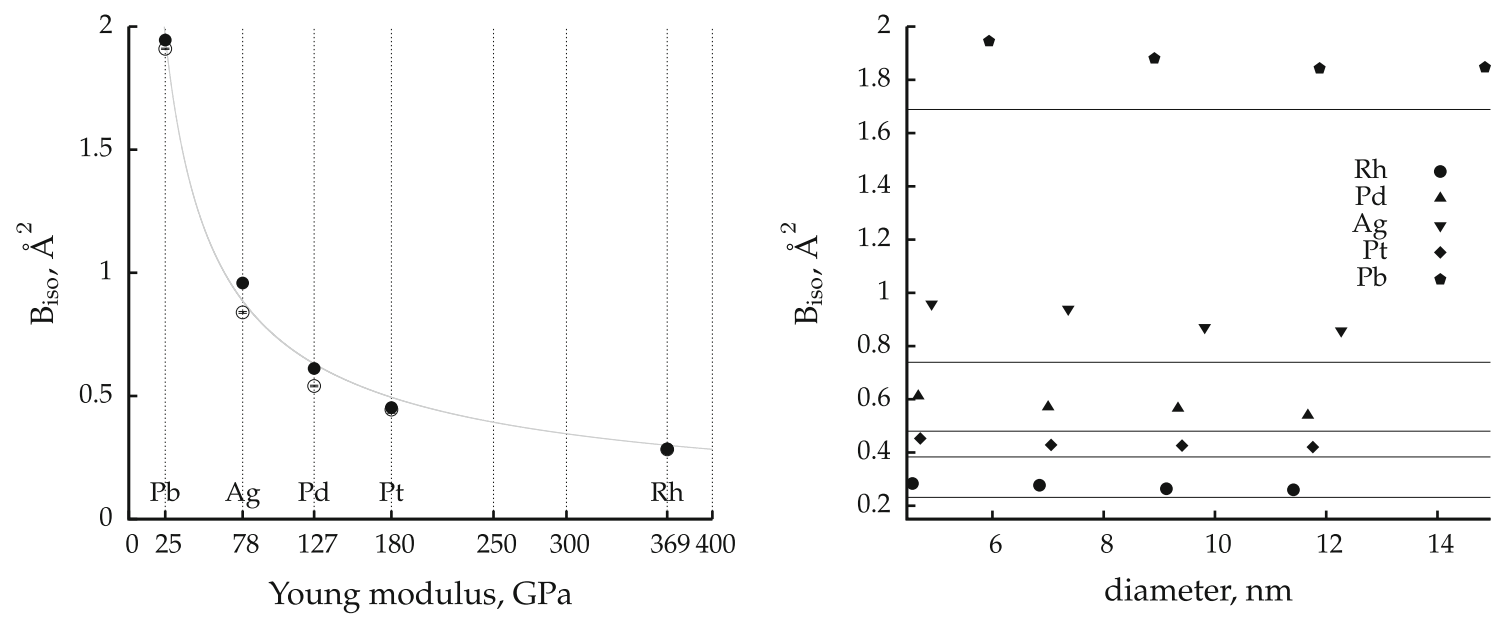

Fig. 4- $B_{\text {iso }}$ as a function of the Young modulus of different metals (left). Values from MD simulations (full symbol, Eq. [3]), and WPPM of time-averaged XRPD data (open symbol). $B_{\text {iso }}$ is also shown as a function of sphere diameter (right). Horizontal lines represent $B_{\text {iso }}$ calculated for a $40 \times 40 \times 40$ unit cell cube, implementing PBCs in the MD simulation.

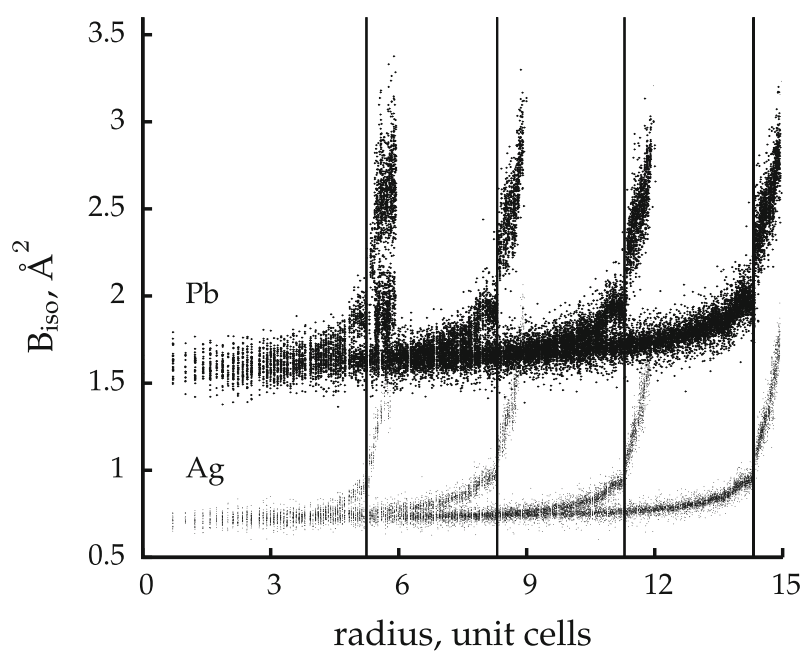

Fig. $5-B_{\text {iso }}$ as a function of the radial coordinate in four spheres with different radius, expressed in number of unit cells for Ag and $\mathrm{Pb}$. Vertical lines mark the threshold above which the number of nearest neighbors decreases below 12 (bulk/surface interface). It is interesting to notice that the behavior of $B_{\text {iso }}$ as a function of the radius predicted by MD is similar for $\mathrm{Ag}$ and $\mathrm{Pd}$.

scattering effect for completely uncorrelated atomic vibrations (left) are shown. ${ }^{[16]}$ In both cases it is quite evident that the TDS effect cannot be ignored. The effect of disregarding it (or of using a poor model like that for uncorrelated vibration), gets worst for (1) decreasing size of the nanocrystal and (2) lower Young modulus, which implies wider oscillations.

Even if most of the peak broadening is caused by the small domain size, local variations in the distance between scattering centers caused by atomic displacement from the ideal (as-built) positions also contribute to alter the line profiles. As explained in the previous paragraph, this complex effect was treated in a simplified way within the WPPM, which although not totally rigorous is sufficiently informative.

Using the results from WPPM for the smallest spheres, Figure 7(a) shows the so-called Warren's plot, ${ }^{[40]}$ i.e., the Root Mean Squared Displacement (RMSD, $\left\langle\Delta L^{2}\right\rangle^{1 / 2}=L\left\langle\varepsilon^{2}\right\rangle^{1 / 2}$ ) as a function of $L$, the distance between couples of scatterers. According to Warren's theory unit cells can be taken as scatterers.

For larger spheres the effect follows similar trends, but with much smaller absolute values. Results refer to an isotropic model, where the invariant form of Miller indices, $A+B H$ is used with $B=0$. The amount of microstrain can be assessed in different ways: based on Figures 7(a) and (b), microstrain and anisotropy range from the lowest level, $\mathrm{Rh}$, corresponding to the largest Young modulus and small Zener ratio, to the highest values, for $\mathrm{Pb}$, which has the smallest Young modulus and highest Zener ratio.

The same analysis can be repeated for each different crystallographic direction taking into account anisotropy $(B \neq 0)$. Two extreme cases for the fcc metals considered, corresponding to the elastically soft and stiff directions ( $h 00$ and $h h h$, respectively), with the isotropic and all other values laying in between, are shown in Figure 8. It is worth noting that, in terms of quality of line profile modeling adding the anisotropy gives a modest (although still significant) improvement. Consistently with the amount of strain and related profile broadening, $\mathrm{Pb}$ and $\mathrm{Rh}$ are again the two extremes (see also Figure 7(b)), with the former the most affected by (macro and micro) strain anisotropy: Goodness of Fit (GoF) improves of 3.33 pct for $\mathrm{Pb}$ and just 0.04 pet for $\mathrm{Rh}$, the other metals laying in between.

As shown thus far, modeling the static disorder resulting from lattice relaxation is not an easy task for RS methods: the complexity of the effect calls for a DS analysis. The left part of Figure 9(a) shows a modeling of a space-averaged sample (Ag, 6 uc radius) using the DSE and Eq. [6], fitting $1, \alpha, a$ and $\sigma$ to displace atoms (see WPPM and DSE). Figure 9(b) shows the radial deformation (Eq. [2]), measured for simulated (spaceaveraged) particle and the object generated by the fitting algorithm (model) as a function of the number of nearest neighbors of a given atom; Figure 9(c) the same quantity as a function of the radial position of a given 

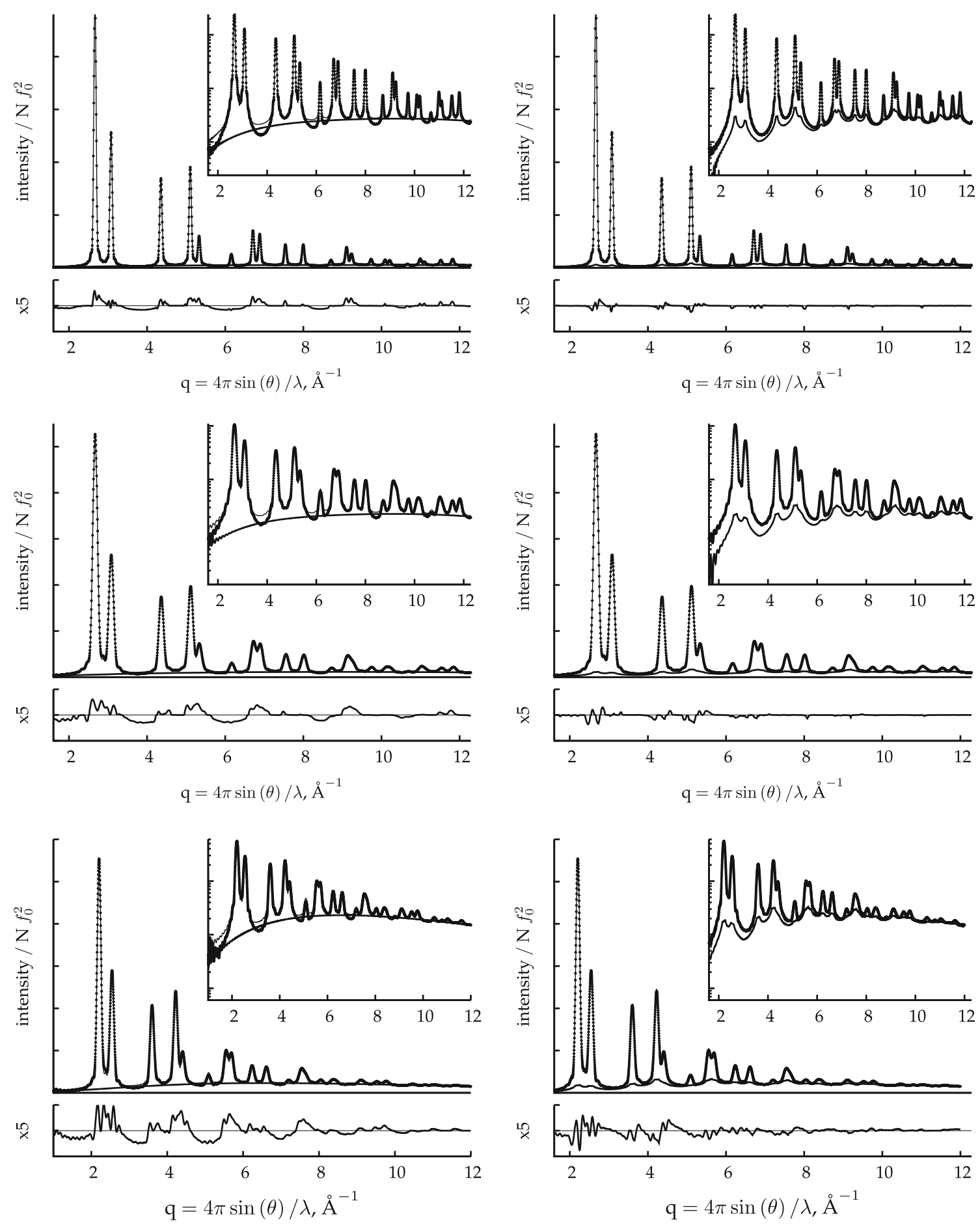

Fig. 6-Modeling results with TDS models based on correlated (right) and uncorrelated (left) atomic vibrations. Spherical domains with different radius (in unit cells, uc), from the top: Ag 12 uc (uncorrelated $N R S S=7.9 \times 10^{-12}$, correlated $N R S S=1.1 \times 10^{-12}$ ), Ag 6 uc (uncorrelated $N R S S=3.9 \times 10^{-10}$, correlated NRSS $=6.4 \times 10^{-11}$ ), and $\mathrm{Pb} 6$ uc (uncorrelated $N R S S=4.1 \times 10^{-10}$, correlated NRSS $=1.0 \times 10^{-10}$ ). The inset shows a logarithmic representation of the same pattern.

atom in its reference (as-built) configuration. This dualview of the quantity underlines the complexity of the displacement field. Particularly, Figure 9(b) illustrates that, although the average value of the strain (circles) is roughly reproduced by the model, the standard deviation (bars) of the strain distribution for a given atomic environment $(Z)$ is underestimated by the model (see Reference 14 for a discussion on the complexity of the strain field). Worst case scenario is illustrated by the smallest $\mathrm{Pb}$ sphere (6 uc radius): the quality of the fit is definitely worse than for the Ag case, but still remarkably good with respect to the RS approach. It is quite 

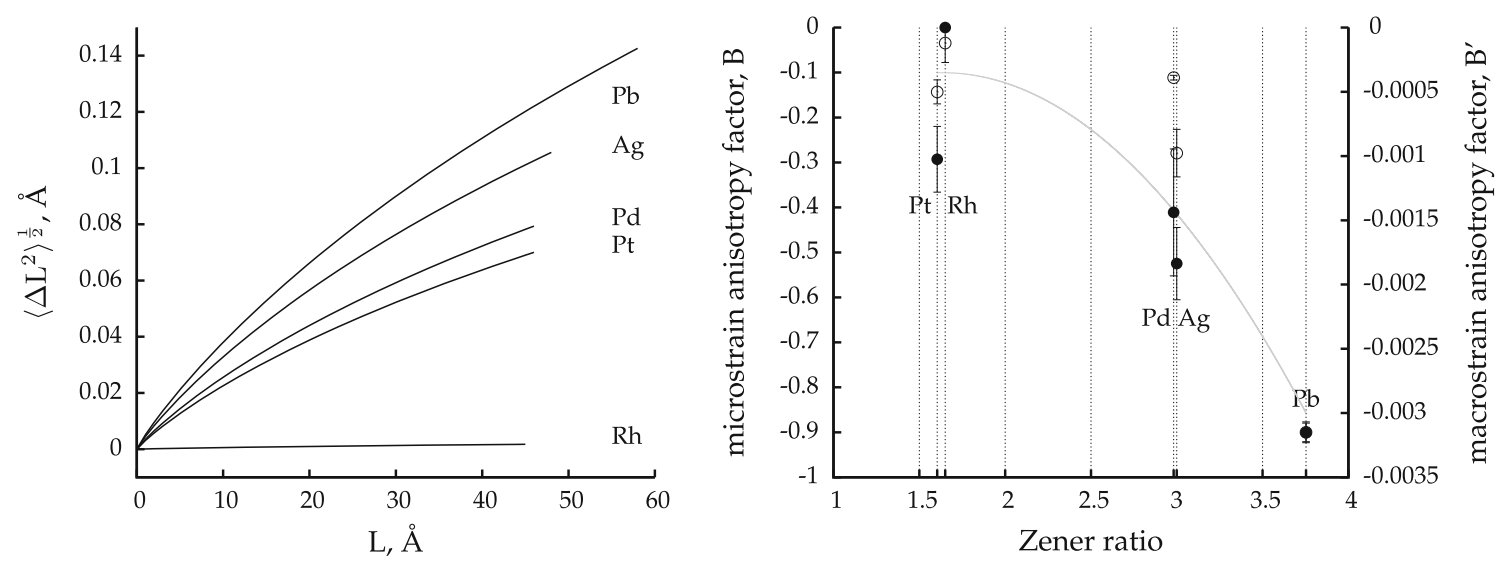

Fig. 7-Warren's plot (left): RMSD as a function of $L$; anisotropy of microstrain affecting line broadening $(B, \bullet)$ and of macrostrain influencing peak position $\left(B^{\prime}, \circ\right)$ as a function of the Zener ratio, which is 1 for the isotropic case (right: the line is drawn just to guide the eye). All values are obtained from WPPM as those shown in Fig. 6 using the TDS model for correlated atomic vibrations.
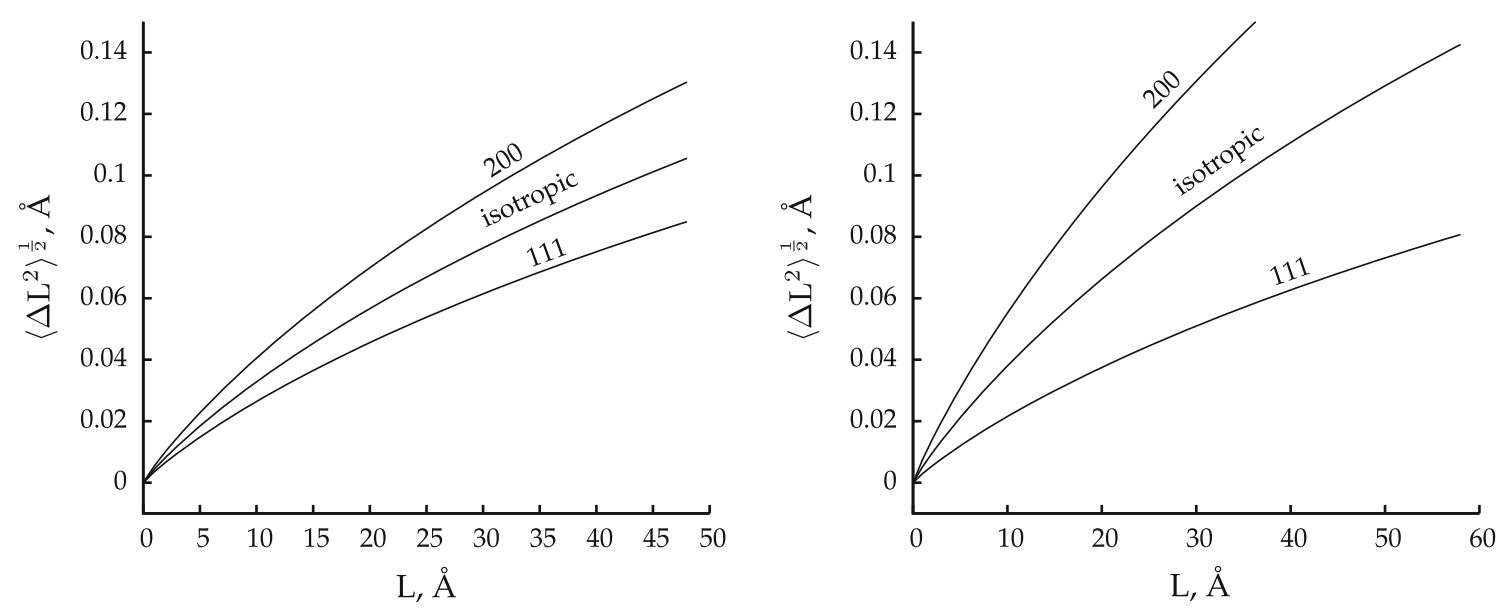

Fig. 8-Warren's plot for $\mathrm{Ag}$ (left) and $\mathrm{Pb}$ (right) spheres (6 uc radius), considering an anisotropic RMSD model (see text for details).

evident from the data in Figure 9 that an analytical model, although based on several functional dependences and adjustable parameters, can hardly reproduce the intricate pattern of atomic displacements caused by the surface relaxation of nanocrystals.

So far only spherical domains have been discussed. Shape of course has also a relevant role, for the effect of bounding the crystal among facets with different crystallographic orientation. As an example, three more geometries are shown, carved out of an infinite $\mathrm{Pb}$ lattice with (1) (100) planes (cube), (2) (110) planes (rhombic dodecahedron) and (3) (111) planes (octahedron). Size was chosen so to include approximately the same number of atoms $(12187,11393$, and 11720, respectively).

The displacement field (Figure 10), which results from the interplay between symmetry of the elastic tensor and of the given object, is quite complicated both in the distribution of amplitude and direction of the effect. The spherical case discussed so far seems to be the simplest one, with the lowest effect of anisotropy, as a result of the averaging effect caused by the higher (spherical) shape symmetry.
Each shape has a different and rather peculiar displacement map, and further differences are to be expected for other elements. Figure 11(a) shows the average deformation for the above-introduced objects. The choice of the abscissa is based on the assumption that deformation is caused by the pressure arising from surface energy at the particle/vacuum interface. This quantity can be expressed by the Young-Laplace equation,

$$
\sigma=2 \frac{\gamma}{r}
$$

and within the limits of an elastic regime (so that Young modulus $E$ and Hooke's law can be used),

$$
\varepsilon=2 \frac{\gamma}{E r} \text {. }
$$

As a consequence, deformation in Figure 11(a) is conveniently shown as a function of $\gamma_{h k l} / E_{h k l}$, the ratio for the crystallographic planes enclosing the nanocrystal. It is worth noting that the spherical case, showing no specific facet, has no abscissa and is conventionally left near the ordinate axis. 

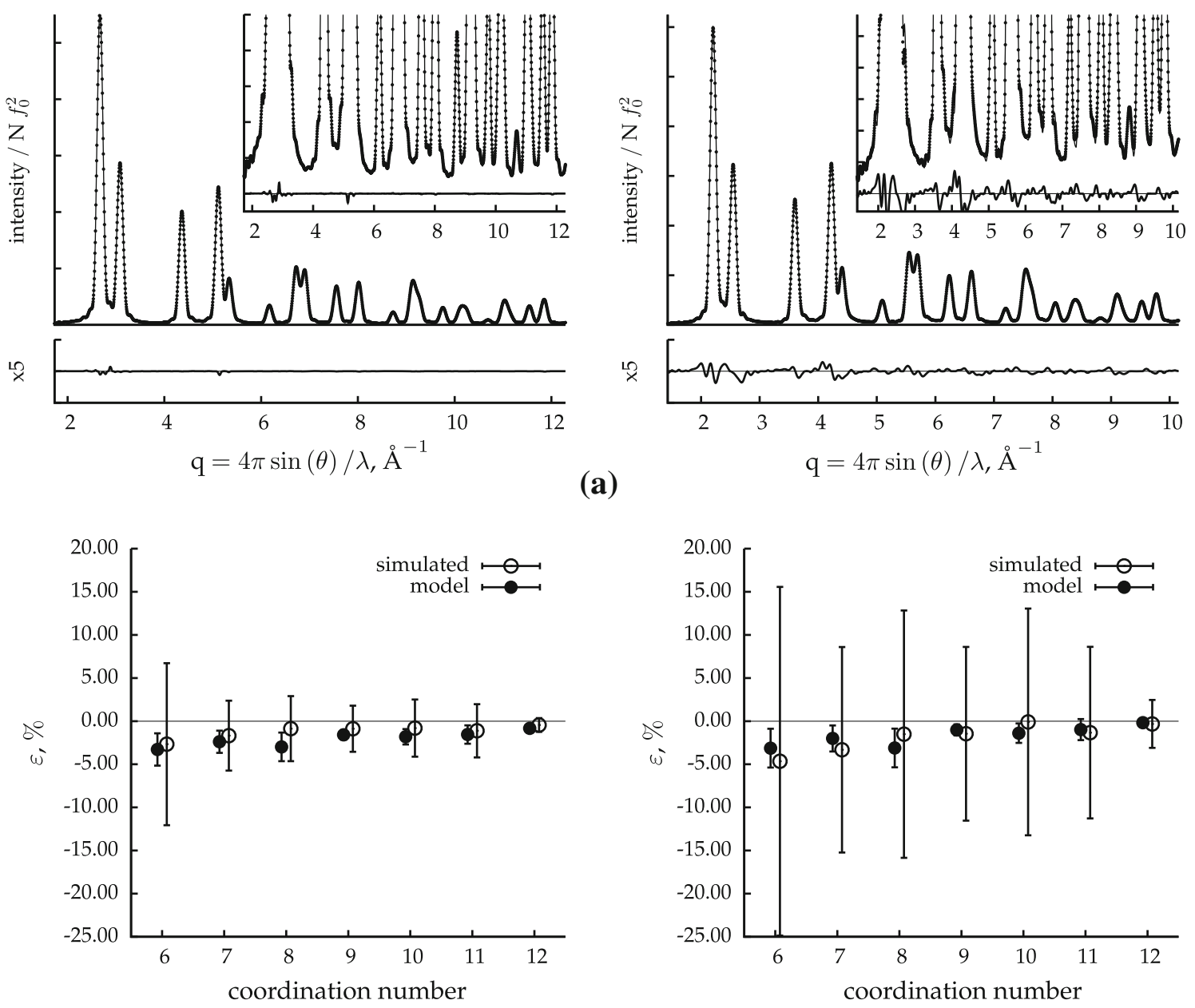

(b)
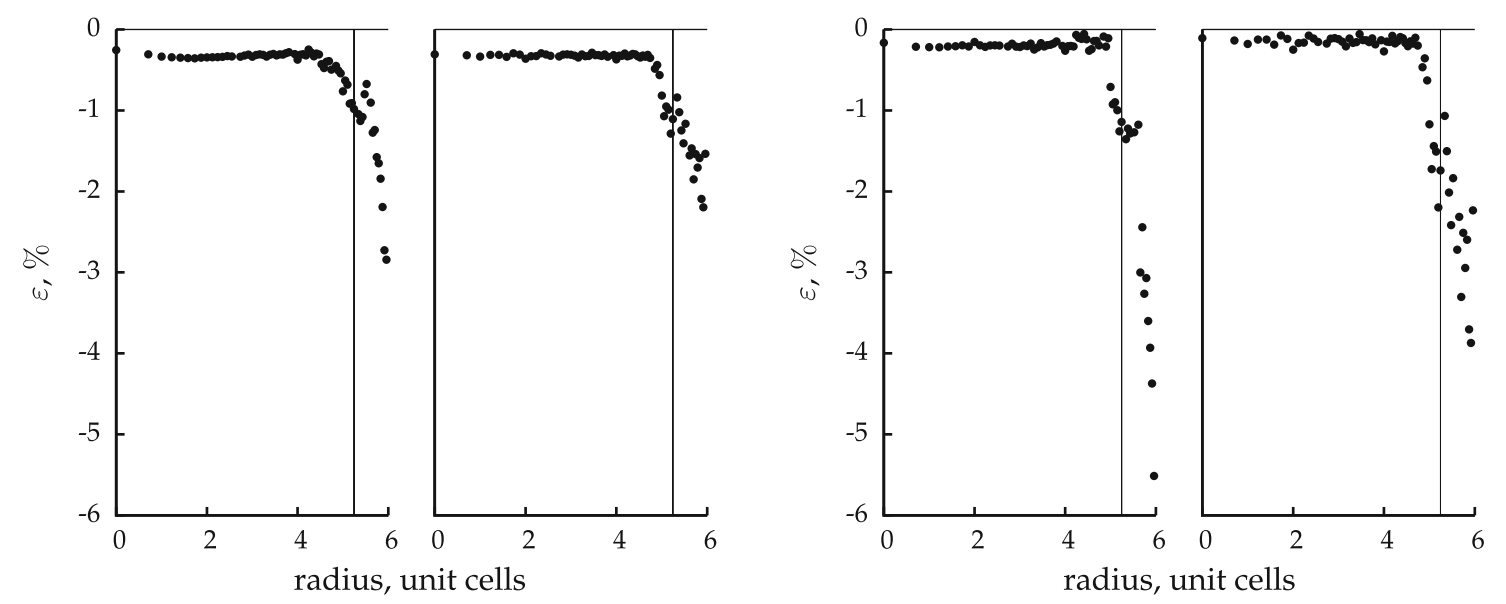

(c)

Fig. 9-Modeling of the space-average XRPD patterns of Ag (left; $N R S S=1.3 \times 10^{-11}$ ) and $\mathrm{Pb}$ (right; $N R S S=4.1 \times 10^{-11}$ ); the inset shows a magnified region of the pattern whereas the residual is shown above. Radial deformation as a function of the numbers of nearest neighbors is also shown $(b)$ : points represent average values, bars standard deviation. $(c)$ Deformation along radial direction. In each figure, curves on the left represent deformation calculated for the space-averaged object, whereas on the right it is shown the result obtained by fitting Eq. [6] to the DSE data. Vertical lines represent the bulk/surface interface.

Figure 11(b) shows $B_{\text {iso }}$ as a function of the Young modulus projected along the direction perpendicular to a given face. The linear relation is justified by the $h k l$ dependence, which is embodied in the expression of the elastic modulus (within the limits of an elastic continuum approximation), 

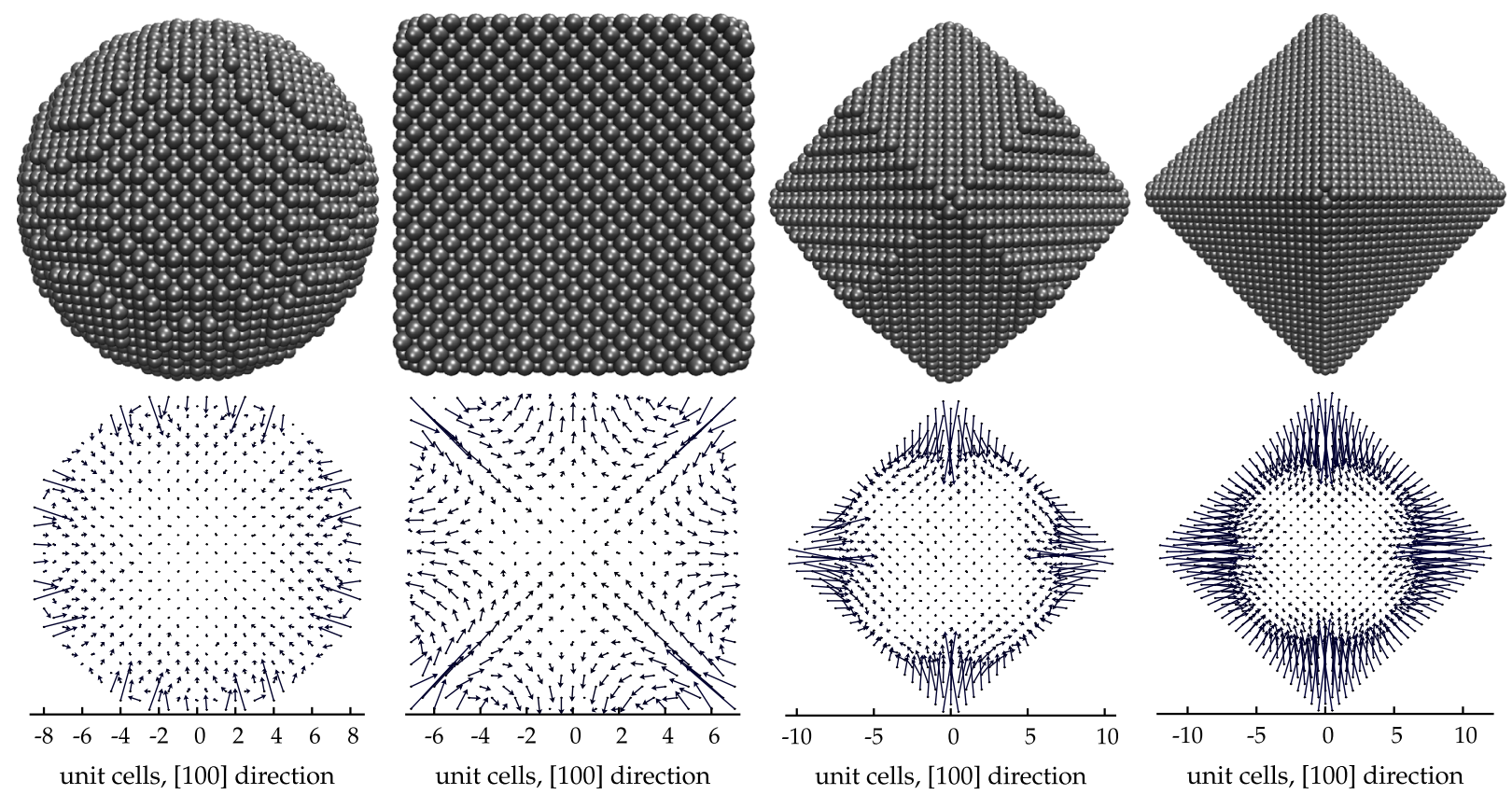

Fig. 10-Displacement maps of four Pb samples. From left to right, sphere, cube, rhombic dodecahedron and octahedron. Maps refer to the (100) cross section (above), with the magnitude of the displacement vector multiplied by 25 times.

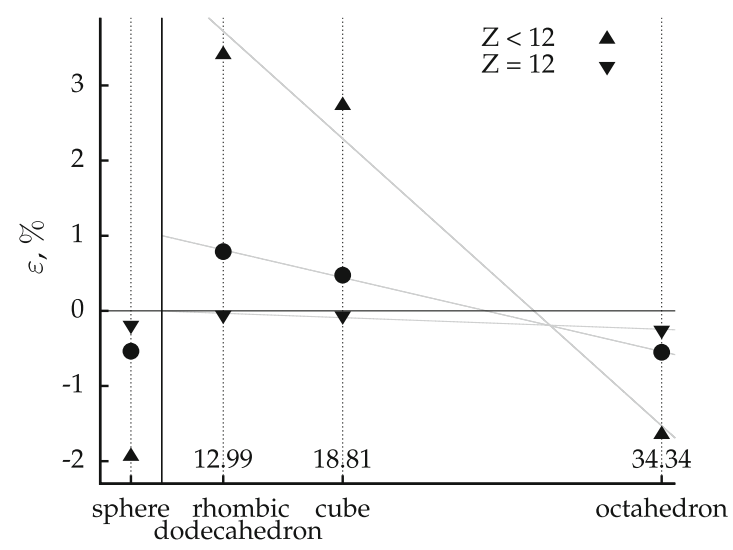

$\gamma_{\mathrm{hkl}} / \mathrm{E}_{\mathrm{hkl}}, \mathrm{pm}$

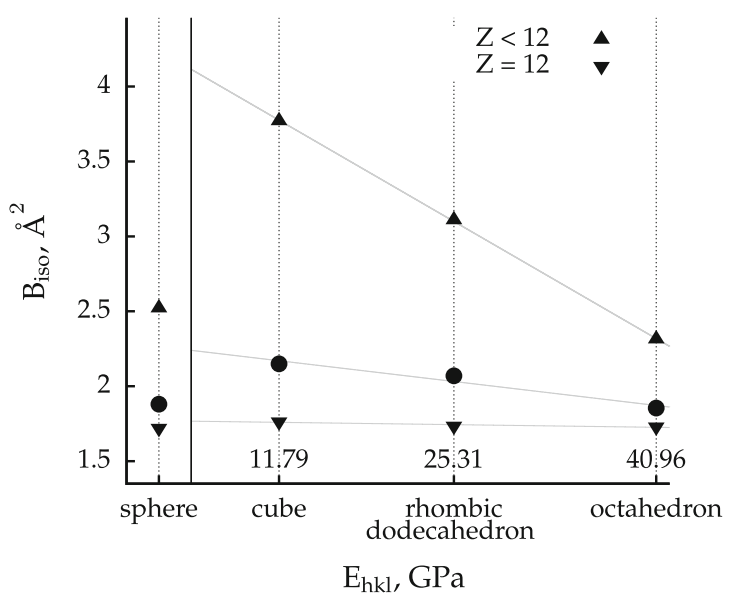

$\mathrm{E}_{\mathrm{hkl}}, \mathrm{GPa}$

Fig. 11-Average strain for systems exposing different $h k l$ as a function of the ratio between surface energy of the facets and elastic modulus normal to the facets (left); $B_{\text {iso }}$ is plotted against elastic modulus along the normal to the facets (right). $Z$ is the atomic coordination, so $Z<12$ $(\mathbf{\Delta})$ is for surface and $Z=12(\boldsymbol{\nabla})$ for bulk atoms, whereas full circle $(\bullet)$ refer to the average value. Lines are drawn to drive the eye.

$$
\frac{1}{E_{h k l}}=S_{11}-2\left[S_{11}-S_{12}-\frac{S_{44}}{2}\right] \frac{h^{2} l^{2}+l^{2} k^{2}+k^{2} h^{2}}{\left(h^{2}+k^{2}+l^{2}\right)^{2}}[10]
$$

This provides again for a dependency on the cubic invariant form ( $c f$. $H$ in WPPM and DSE), so that the softest [h00] direction allows the widest out-of-plane atomic oscillations perpendicular to cube faces, whereas the stiffest $[h h h]$ gives the narrowest oscillations normal to the octahedron faces, with the rhombic dodecahedron (framed by $\{h h 0\}$ ) laying in between. As in Figure 11(a), the sphere has no given abscissa, and corresponding values fall in between the limits of the [h00] and $[h h h]$ cases. It is also worth noting that the anisotropy effect is much stronger for less bounded atoms, i.e., those with a coordination lower than 12 .

\section{CONCLUSIONS}

Nanocrystals cannot be simply represented as deformed small crystals: analytical models suggested by continuum mechanics, which are rigorous for materials at the macro-scale, may be a reasonable first-order approximation, but cannot explain the fine details and complex effects influencing the atomic arrangement. Atomistic models can address a proper description of real nanocrystals, allowing to understand and interpret the complexity of the static and dynamic properties. 
This approach naturally calls for a DS representation of diffraction, which is as detailed as computationally heavy: for this reason a traditional RS approach, much faster to implement and use, can still provide useful information, provided that thermal effects are properly considered, together with a suitable representation of the elastic anisotropy effects influencing both peak position and broadening. It is worth considering that the complexity of nanocrystals and many specific properties of interest are strongly influenced by their shape: nanocrystals framed by different crystallographic planes deeply differ in properties, and the modeling of the powder diffraction pattern might be definitely too difficult to be viable for traditional RS methods.

\section{REFERENCES}

1. C. Hsu, C. Huang, Y. Hao, and F. Liu: Nanoscale Res. Lett., 2013, vol. 8, pp. 113-20.

2. R. Long, K. Mao, X. Ye, W. Yan, Y. Huang, J. Wang, Y. Fu, X. Wang, X. Wu, Y. Xie, and Y. Xiong: J. Am. Chem. Soc., 2013, vol. 135 , pp. $3200-07$.

3. A.X. Yin, X.Q. Min, W. Zhu, H.S. Wu, Y.W. Zhang, and C.H. Yan: Chem. Commun., 2012, vol. 48, pp. 543-45.

4. J. Wu, L. Qi, H. You, A. Gross, J. Li, and H. Yang: J. Am. Chem. Soc. , 2012, vol. 134, pp. 11880-83.

5. L. Gan, R. Yu, J. Luo, Z. Cheng, and J. Zhu: J. Phys. Chem. Lett., 2012, vol. 3, pp. 934-38.

6. C. Cui, L. Gan, M. Heggen, S. Rudi, and P. Strasser: Nat. Mater., 2013, vol. 12, pp. 765-71.

7. T. Fujita, P. Guan, K. McKenna, X. Lang, A. Hirata, L. Zhang, T. Tokunaga, S. Arai, Y. Yamamoto, N. Tanaka, Y. Ishikawa, N. Asao, Y. Yamamoto, J. Erlebacher, and M. Chen: Nat. Mater., 2012, vol. 11, pp. 775-80.

8. P. Scardi, M. Leoni, and R. Delhez: J. Appl. Crystallogr., 2004, vol. 37, pp. 381-90.

9. E.J. Mittemeijer and P. Scardi, eds.: Diffraction Analysis of Materials Microstructure, vol. 68 of Springer Series in Materials Science, Springer, Berlin, 2004.

10. P. Scardi: Powder Diffraction: Theory and Practice, Chap. XIII, The Royal Society of Chemistry, Cambridge, U.K., 2008, pp. 376 413.

11. B.D. Hall: J. Appl. Phys., 2000, vol. 87, pp. 1666-75.

12. A. Cervellino, C. Giannini, and A. Guagliardi: J. Appl. Crystal$\operatorname{logr} ., 2003$, vol. 36, pp. 1148-58.
13. L. Gelisio, C.L. Azanza Ricardo, M. Leoni, and P. Scardi: J. Appl. Crystallogr., 2010, vol. 43, pp. 647-53.

14. L. Gelisio, K.R. Beyerlein, and P. Scardi: Thin Solid Films, 2013, vol. 530, pp. 35-39

15. P. Debye: Ann. Phys., 1915, vol. 351, pp. 809-23.

16. B.E. Warren: X-Ray Diffraction, Dover, New York, 1990

17. L. Gelisio, C.L. Azanza Ricardo, M. Leoni, and P. Scardi: Zeitschrift für Kristallographie Proceedings, 2011, vol. 1, pp. 18994.

18. S. Plimpton: J. Comput. Phys., 1995, vol. 117, pp. 1-19.

19. M.S. Daw and M.I. Baskes: Phys. Rev. Lett., 1983, vol. 50, pp. $1285-88$.

20. M.S. Daw and M.I. Baskes: Phys. Rev. B, 1984, vol. 29, pp. $6443-$ 53.

21. H.W. Sheng, M.J. Kramer, A. Cadien, T. Fujita, and M.W. Chen: Phys. Rev. B, 2011, vol. 83, pp. 134118-137.

22. P. Scardi and M. Leoni: Acta Crystallogr. Sect. A, 2001, vol. 57, pp. 604-13.

23. P. Scardi and M. Leoni: Acta Crystallogr. Sect. A, 2002, vol. 58, pp. $190-200$

24. A. Leonardi, M. Leoni, S. Siboni, and P. Scardi: J. Appl. Crystallogr., 2012, vol. 45, pp. 1162-72.

25. J. Martinez-Garcia, M. Leoni, and P. Scardi: Acta Crystallogr. Sect. A, 2009, vol. 65, pp. 109-19.

26. K.R. Beyerlein, R.L. Snyder, and P. Scardi: J. Appl. Crystallogr., 2011, vol. 44, pp. 945-53.

27. M. Leoni and P. Scardi: Diffraction Analysis of the Microstructure of Materials, Chap. XVI, pp. 413-52, Springer, Berlin, 2004.

28. J.P. Urban: Acta Crystallogr. Sect. A, 1975, vol. 31, pp. 95-100.

29. K.R. Beyerlein, M. Leoni, and P. Scardi: Acta Crystallogr. Sect. A, 2012, vol. 68, pp. 382-92.

30. T. Adler and C.R. Houska: J. Appl. Phys., 1979, vol. 50, pp. 3282 87.

31. N.C. Popa: J. Appl. Crystallogr., 1998, vol. 31, pp. 176-80.

32. K.R. Beyerlein, R.L. Snyder, M. Li, and P. Scardi: J. Nanosci. Nanotechnol., 2012, vol. 12, pp. 8554-60.

33. L. Gelisio and P. Scardi: J. Nanosci. Nanotechnol., 2012, vol. 12, pp. 1-7.

34. T. Ino and N. Minami: Acta Crystallogr. Sect. A, 1984, vol. 40, pp. 538-44.

35. C.C. Yang, M.X. Xiao, W. Li, and Q. Jiang: Solid State Commun., 2006, vol. 139, pp. 148-52.

36. R. Kumar, G. Sharma, and M. Kumar: J. Thermodyn., 2013, vol. 2013, pp. 328051-55.

37. R. Meyer and P. Entel: Z. Kristallogr., 2007, vol. 11, pp. 646-49.

38. Y.H. Zhao, H.W. Sheng, and K. Lu: Acta Mater., 2001, vol. 49, pp. 365-75.

39. L.M. Peng, G. Ren, S.L. Dudarev, and M.J. Whelan: Acta Crystallogr. Sect. A, 1996, vol. 52, pp. 456-70.

40. B.E. Warren and B.L. Averbach: J. Appl. Phys., 1950, vol. 21, pp. 595-99. 